\title{
Dynamic Analysis and Structure Soil Interaction of Retaining Wall Using ETABS
}

\author{
T.Subramani ${ }^{1 *}$, E.Narendra Kumar ${ }^{2}$ \\ $1^{*}$ Managing Director, Priyanka Associates (Civil Engineers and Valuers), Salem, TamilNadu, India. \\ ${ }^{2}$ Design and Site Engineer, M/s.Amman Associates (Civil Engineers and Valuers), Salem, TamilNadu, India. \\ Corresponding author E-mail:tsmcivil2007@gmail.com
}

\begin{abstract}
Retaining systems are widely used international for serving numerous functions in structures and infrastructures. The seismic response of forms of walls that assist a single soil layer has been examined with the aid of some of researchers in the past. The design of preserving partitions in seismic areas poses a complex problem. The conventional layout method usually contains calculation of an element of safety in opposition to sliding, overturning and bearing ability failure. Retaining partitions have suffered damages under beyond earthquakes. Typically the analyses do not bear in mind the retained soil's interplay with the wall, which takes location at some point of dynamic conditions. The situations of separation of wall (at some point of interactions) over again trade the dynamic traits of the assumed wallsoil interplay that needs to be addressed. Our study conducts the retaining wall beneath static in addition to seismic situations about above components.
\end{abstract}

Keywords: Dynamic Analysis, Soil Interaction, Retaining wall and ETABS

\section{Introduction}

Seismic damage to gravity wall results from basis failure due to excessive plastic floor deformation. accordingly the harm of a wall is especially associated with the motion and failure brought approximately through excessive seismic earth strain. but, the to be had models within the literature were now not without a doubt completed an excellent manner to are expecting the seismic response of the wall all through earthquake.

\section{Methodology}

Fig. 1 shows the methodology of this study.

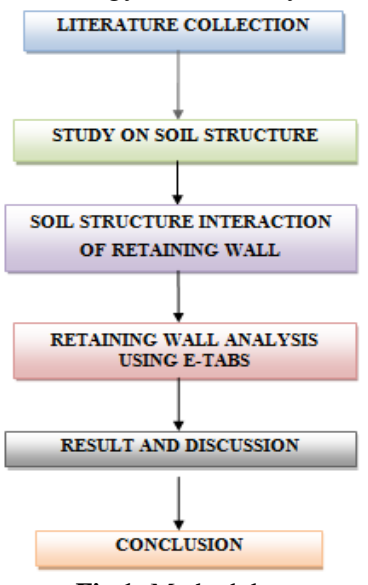

Fig.1: Methodology

\section{Retaining Wall}

Techniques for comparing earth pressures on maintaining walls and design techniques are summarized herein for cohesion much less backfill materials, which must be used each time viable. Earth keeping structures are designed to triumph over significant in ground tiers to offer both a sloping or flat floor at the retained face. Earth maintaining structures can help provision of feasible vicinity for extraordinary civil engineering systems to be built.

\section{Earthquake}

\subsection{Basic Aspects of Seismic Design}

Designing homes to act elastically at some point of earthquakes without harm can also render the mission economically unviable. Accordingly, it may be vital for the structure to undergo harm and thereby dissipate the electricity input to it all through the earthquake.

- $\quad$ Slight shaking with minor damage to structural elements, and a few damage to non-structural factors; and

- Thus, seismic layout balances reduced price and ideal harm, to make the task viable. This cautious balance is arrived primarily based on giant research and detailed put upearthquake damage assessment research.

- A wealth of this record is translated into specific seismic layout provisions. In assessment, structural damage isn't acceptable beneath layout wind forces. Because of this, layout in opposition to earthquake results is referred to as 
earthquake-resistant layout and now not earthquake-proof design.

\subsection{Earthquake Resistant Structures - Factors}

- All elements consisting of partitions and the roof must be tied collectively on the way to act as an included unit all through earthquake shaking.

- The constructing have to be linked to an amazing quality and the earth moist soil have to be avoided and the inspiration need to be nicely tied collectively as nicely tied to the wall wherein the soft soils strengthening must be provided.

\section{Analysis Software}

\subsection{E-Tabs}

ETABS is a complicated, however smooth to apply, unique motive evaluation and layout application superior specifically for constructing structures.

\section{Analysis Results}

\subsection{Structure Data}

This area presents model geometry statistics, inclusive of items consisting of story levels, point coordinates, and detail connectivity. Fig. 2 suggests the summary report.

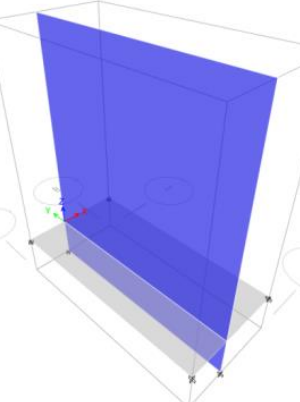

Fig.2: Summary report

\subsubsection{Storey Data}

Table 1 shows the storey data.

Table 1: Storey data

\begin{tabular}{|c|c|c|c|c|c|}
\hline Name & $\begin{array}{c}\text { Height } \\
\mathbf{m m}\end{array}$ & $\begin{array}{c}\text { Elevation } \\
\mathbf{m m}\end{array}$ & $\begin{array}{c}\text { Master } \\
\text { Story }\end{array}$ & $\begin{array}{c}\text { Similar } \\
\text { To }\end{array}$ & $\begin{array}{c}\text { Splice } \\
\text { Story }\end{array}$ \\
\hline Story 2 & $\mathbf{5 0 0 0}$ & $\mathbf{5 0 0 0}$ & No & None & No \\
\hline Storyl & 1000 & 0 & No & None & No \\
\hline Base & 0 & -1000 & No & None & No \\
\hline
\end{tabular}

\subsection{Loads}

\subsubsection{Load Patterns}

Table 2 shows the load patterns.

Table 2: Load Patterns

\begin{tabular}{|c|c|c|}
\hline Name & Type & $\begin{array}{c}\text { Self Weight } \\
\text { Multiplier }\end{array}$ \\
\hline 1 LOAD CASE 1 & Dead & 1.5 \\
\hline 2 LOAD CASE 2 & Reducible Live & 0 \\
\hline
\end{tabular}

\subsubsection{Load cases}

Table 3 shows the load cases - summary.

Table 3: Load cases - summary

\begin{tabular}{|c|c|}
\hline Name & Type \\
\hline Dead & Linear Static \\
\hline Live & Linear Static \\
\hline 1 LOAD CASE 1 & Linear Static \\
\hline 2 LOAD CASE 2 & Linear Static \\
\hline
\end{tabular}

\section{Analysis Results}

\subsection{Structure Results}

Table 4 shows the base reactions.

Table 4: Base Reactions

\begin{tabular}{|c|c|c|c|c|c|c|c|c|c|}
\hline $\begin{array}{c}\text { Load } \\
\text { Case/Comb } \\
\mathbf{0}\end{array}$ & $\begin{array}{c}\mathrm{FX} \\
\mathrm{kN}\end{array}$ & $\begin{array}{c}\mathrm{FY} \\
\mathrm{kN}\end{array}$ & $\begin{array}{c}\mathrm{FZ} \\
\mathrm{kN}\end{array}$ & $\begin{array}{c}\mathrm{MX} \\
\mathrm{kN}-\mathrm{m}\end{array}$ & $\begin{array}{c}\mathrm{MY} \\
\mathrm{kN}-\mathrm{m}\end{array}$ & $\begin{array}{c}\mathrm{MZ} \\
\mathrm{kN}-\mathrm{m}\end{array}$ & $\begin{array}{c}\mathrm{X} \\
\mathrm{m}\end{array}$ & $\begin{array}{c}\mathrm{Y} \\
\mathrm{m}\end{array}$ & $\begin{array}{c}\mathrm{Z} \\
\mathrm{m}\end{array}$ \\
\hline Dead & 0 & 0 & 0 & 0 & 0 & 0 & 0 & 0 & -1 \\
\hline Live & 0 & 0 & 0 & 0 & 0 & 0 & 0 & 0 & -1 \\
\hline $\begin{array}{c}\text { 1LOAD } \\
\text { CASE 1 }\end{array}$ & 0 & 0 & 305.2171 & -763.0428 & -22.4424 & 0 & 0 & 0 & -1 \\
\hline $\begin{array}{c}\text { 2 LOAD } \\
\text { CASE 2 }\end{array}$ & $\begin{array}{c}175.78 \\
13\end{array}$ & 0 & 37.5 & -93.75 & 108.5938 & -439.4533 & 0 & 0 & -1 \\
\hline
\end{tabular}

Table 5 shows the centres of mass and rigidity.

Table 5: Centres of mass and rigidity

\begin{tabular}{|c|c|c|c|c|c|c|c|c|c|}
\hline Story & $\begin{array}{c}\text { Diaphr } \\
\text { agm }\end{array}$ & $\begin{array}{c}\text { Mass } \\
\mathbf{k g}\end{array}$ & $\begin{array}{c}\text { Mass } \\
\mathbf{~ k g}\end{array}$ & $\begin{array}{c}\text { XCM } \\
\mathrm{m}\end{array}$ & $\begin{array}{c}\text { YCM } \\
\mathrm{m}\end{array}$ & $\begin{array}{c}\text { Cumul } \\
\text { ative X } \\
\mathrm{kg}\end{array}$ & $\begin{array}{c}\text { Cumul } \\
\text { ative Y } \\
\mathrm{kg}\end{array}$ & $\begin{array}{c}\text { XCC } \\
\mathbf{M} \\
\mathrm{m}\end{array}$ & $\begin{array}{c}\text { YCC } \\
\mathbf{M} \\
\mathrm{m}\end{array}$ \\
\hline Storyl & $\mathrm{Dl}$ & $\begin{array}{c}13425 . \\
82\end{array}$ & $\begin{array}{c}13425 \\
82\end{array}$ & 0.1136 & $-\mathbf{2 . 5}$ & $\begin{array}{c}13425 . \\
82\end{array}$ & $\begin{array}{c}13425 . \\
82\end{array}$ & 0.1136 & -2.5 \\
\hline
\end{tabular}

\subsection{Modal Results}

Table 6 shows the model periods and frequencies.

Table 6: Model periods and frequencies

\begin{tabular}{|c|c|c|c|c|c|}
\hline Case & Mode & $\begin{array}{c}\text { Period } \\
\mathrm{sec}\end{array}$ & $\begin{array}{c}\text { Frequency } \\
\mathrm{cyc} / \mathrm{sec}\end{array}$ & $\begin{array}{c}\text { Circular Frequency } \\
\mathrm{rad} / \mathrm{sec}^{-}\end{array}$ & $\begin{array}{c}\text { Eigenvalue } \\
\mathrm{rad}^{2} / \mathrm{sec}^{2}\end{array}$ \\
\hline Modal & 1 & 11682.376 & $8.56 \mathrm{E}-05$ & 0.0005 & 0 \\
\hline Modal & 2 & 7012.451 & 0.0001426 & 0.0009 & $8.028 \mathrm{E}-07$ \\
\hline Modal & 3 & 101.282 & 0.01 & 0.062 & 0.0038 \\
\hline Modal & 4 & 26.628 & 0.038 & 0.236 & 0.0557 \\
\hline
\end{tabular}

Table 7 shows the mass ratios (part 1 of 2).

Table 7: Mass ratios (Part 1 of 2)

\begin{tabular}{|c|c|c|c|c|c|c|c|c|}
\hline Case & Mode & $\begin{array}{c}\text { Period } \\
\text { sec }\end{array}$ & UX & UY & UZ & $\begin{array}{c}\text { Sum } \\
\text { UX }\end{array}$ & $\begin{array}{c}\text { Sum } \\
\text { UY }\end{array}$ & $\begin{array}{c}\text { Sum } \\
\text { UZ }\end{array}$ \\
\hline Modal & 1 & 11682.376 & 1 & 0 & 0 & 1 & 0 & 0 \\
\hline Modal & 2 & 7012.451 & 0 & 0 & 0 & 1 & 0 & 0 \\
\hline Modal & 3 & 101.282 & 0 & 0.0298 & 0 & 1 & 0.0298 & 0 \\
\hline Modal & 4 & 26.628 & 0 & 0.9702 & 0 & 1 & 1 & 0 \\
\hline
\end{tabular}

Table 8 shows the mass ratios (part 2 of 2). 
Table 8: Mass ratios (Part 2 of 2)

\begin{tabular}{|c|c|c|c|c|c|c|c|}
\hline Case & Mode & $\mathbf{R X}$ & $\mathrm{RY}$ & $\mathbf{R Z}$ & $\begin{array}{c}\text { Sum } \\
\mathrm{RX}\end{array}$ & $\begin{array}{c}\text { Sum } \\
\mathrm{RY}\end{array}$ & $\begin{array}{c}\text { Sum } \\
\mathrm{RZ}\end{array}$ \\
\hline Modal & $\mathbf{1}$ & 0 & 1 & 0 & 0 & 1 & 0 \\
\hline Modal & 2 & 0 & 0 & 0.9991 & 0 & 1 & 0.9991 \\
\hline Modal & 3 & 0.0298 & 0 & $2.58 \mathrm{E}-05$ & 0.0298 & 1 & 0.9992 \\
\hline Modal & 4 & 0.9702 & 0 & 0.0008 & 1 & 1 & 1 \\
\hline
\end{tabular}

Table 9 shows the modal load participation ratios.

Table 9: Modal load participation ratios

\begin{tabular}{|c|c|c|c|c|}
\hline Case & Item Type & Item & Static \% & Dynamic \% \\
\hline Modal & Acceleration & UX & 100 & 100 \\
\hline Modal & Acceleration & UY & 243078219 & 100 \\
\hline Modal & Acceleration & UZ & 0 & 0 \\
\hline
\end{tabular}

Table 10 shows the model direction factors.

Table 10: Model direction factors

\begin{tabular}{|c|c|c|c|c|c|c|}
\hline Case & Mode & $\begin{array}{c}\text { Period } \\
\text { sec }\end{array}$ & UX & UY & UZ & RZ \\
\hline Modal & 1 & 11682.376 & 1 & 0 & 0 & 0 \\
\hline Modal & 2 & 7012.451 & 0 & 0 & 0 & 1 \\
\hline Modal & 3 & 101.282 & 0 & 1 & 0 & 0 \\
\hline Modal & 4 & 26.628 & 0 & 1 & 0 & 0 \\
\hline
\end{tabular}

Fig. 3 shows the deformation.

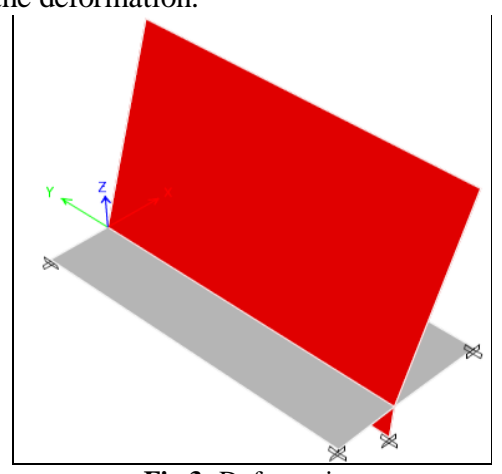

Fig.3: Deformation

Fig.4 shows the stress diagram.

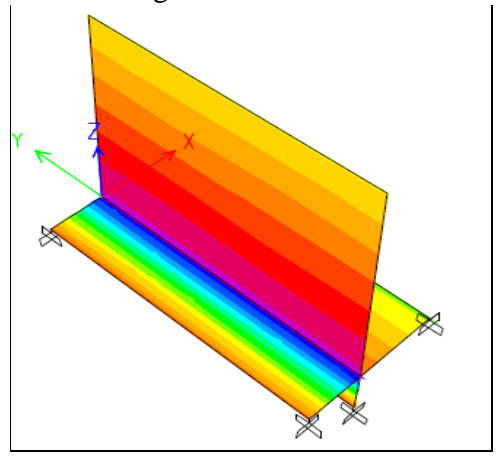

Fig.4: Stress diagram

\subsection{Response Spectrum Analysis}

Fig. 5 shows the spectral acceleration.

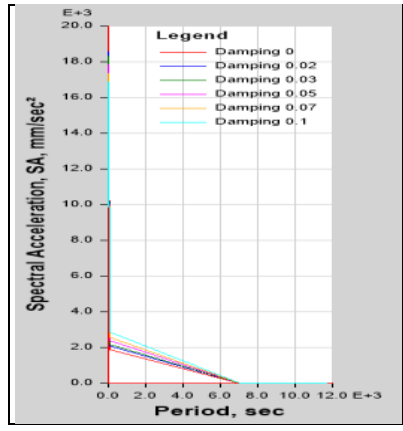

Fig.5: Spectral acceleration

Fig.6 shows the spectral velocity.

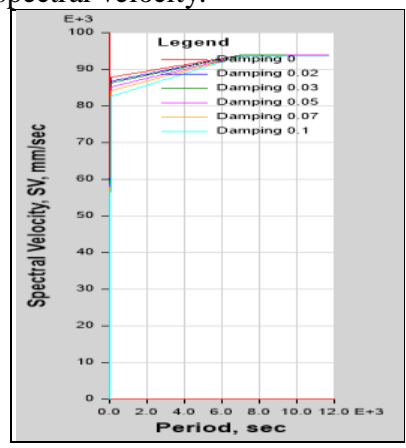

Fig.6: Spectral velocity

Fig.7 shows the time history plot.

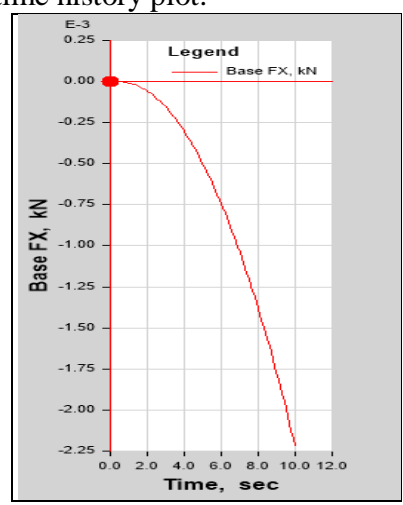

Fig.7: Time history plot

\section{Conclusion}

In the present study at, the usage of two - dimensional numerical simulations, they have an effect on of the wall compliance, the form of the wall-retained soil interface, and the relative peak of the structure at the dynamic misery and response of the latter is tested. During a seismic occasion it is glaring that the dynamic response of each element of this complex tool. Regardless of the fact that there exist many open problems to be resolved,. This mission based on have a look at of earth quake analysis and soil interplay of keeping wall the usage of E-Tabs.

\section{References}

[1]. T.Subramani, J.Jayalakshmi , " Analytical Investigation Of Bonded Glass Fibre Reinforced Polymer Sheets With Reinforced Concrete Beam Using Ansys", International Journal of Application or Innovation in Engineering \& Management (IJAIEM), Volume 4, Issue 5, pp. 105-112, 2015

[2]. T.Subramani. ,S.Vishnupriya, "Finite Element Analysis of a Natural Fiber (Maize) Composite Beam", International Journal of Modern Engineering Research, Volume. 4, Issue. 6 (Version 1), pp $1-7,2014$,

[3]. T.Subramani.,R.Senthil Kumar, "Modelling and Analysis of Hybrid Composite Joint Using Fem in ANSYS", International Journal of 
Modern Engineering Research, Volume 4, Issue 6 (Version 1), pp 41- 46, 2014.

[4]. T.Subramani, S.Sharmila, "Prediction of Deflection and Stresses of Laminated Composite Plate with Artificial Neural Network Aid", International Journal of Modern Engineering Research, Volume 4, Issue 6 (Version 1), pp $51-58,2014$.

[5]. T.Subramani.,S.Sundar, M.Senthilkumar, "Investigation of the Behaviour for Reinforced Concrete Beam Using Non Linear Three Dimensional Finite Elements", International Journal of Modern Engineering Research, Volume. 4, Issue. 6 (Version 2), pp 13 -18, 2014 ,

[6]. T.Subramani,A.Arul, "Design And Analysis Of Hybrid Composite Lap Joint Using Fem" International Journal of Engineering Research and Applications, Volume. 4, Issue. 6 (Version 5), pp 289- 295, 2014.

[7]. T.Subramani., J.Jothi,, M.Kavitha"Earthquake Analysis Of Structure By Base Isolation Technique In SAP", International Journal of Engineering Research and Applications, Volume. 4, Issue. 6 (Version 5), pp 296 - 305, 2014.

[8]. T.Subramani., R.Manivannan.R, M.Kavitha, "Crack Identification In Reinforced Concrete Beams Using Ansys Software" ,International Journal of Engineering Research and Applications, Volume. 4, Issue. 6 (Version 6), pp 133 - 141, 2014.

[9]. T.Subramani.,Reni Kuruvilla, J.Jayalakshmi., "Nonlinear Analysis Of Reinforced Concrete Column With Fiber Reinforced Polymer Bars" International Journal of Engineering Research and Applications Volume. 4, Issue. 6 (Version 5), pp 306- 316, 2014.

[10]. T.Subramani, D.Sakthi Kumar, S.Badrinarayanan. "Fem Modelling And Analysis Of Reinforced Concrete Section With Light Weight Blocks Infill " International Journal of Engineering Research and Applications, Volume. 4, Issue. 6 (Version 6), pp 142 - 149, 2014.

[11]. T.Subramani, B.Saravanan., J.Jayalakshmi., "Dynamic Analysis Of Flanged Shear Wall Using Staad Pro", International Journal of Engineering Research and Applications, Volume. 4, Issue. 6 (Version 6), pp 150 - 155, 2014.

[12]. T.Subramani, M.Subramani., K.Prasath.,"Analysis Of Three Dimensional Horizontal Reinforced Concrete Curved Beam Using Ansys" International Journal of Engineering Research and Applications, Volume. 4, Issue. 6 (Version 6), pp 156 - 161, 2014.

[13]. T.Subramani.,K.Bharathi Devi., M.S.Saravanan. , Suboth ,Analysis Of RC Structures Subject To Vibration By Using Ansys," International Journal of Engineering Research and Applications Vol. 4, Issue 12(Version 5), pp.45-54, 2014.

[14]. T.Subramani., K.Bharathi Devi., M.S.Saravanan., Suboth Thomas, "Analysis Of SeismicPerformanceOfRockBlockStructures With STAAD Pro International Journal of Engineering Research and Applications Vol. 4, Issue 12(Version 5), pp.55- 68, 2014.

[15]. T.Subramani., T.Krishnan., M.S.Saravanan.M , Suboth Thomas, "Finite Element Modeling On Behaviour Of Reinforced Concrete Beam Column Joints Retrofitted With CFRP Sheets Using Ansys" International Journal of Engineering Research and Applications Vol. 4, Issue 12(Version 5), pp.69 -76, 2014

[16]. T.Subramani., S.Krishnan., M.S.Saravanan.M, Suboth Thomas "Analysis Of Retrofitting Non-Linear Finite Element Of RCC Beam And Column Using Ansys" International Journal of Engineering Research and Applications ,Vol. 4, Issue 12(Version 5), pp.77-87, 2014

[17]. T.Subramani, J.Jayalakshmi , " Analytical Investigation Of Bonded Glass Fibre Reinforced Polymer Sheets With Reinforced Concrete Beam Using Ansys" , International Journal of Application or Innovation in Engineering \& Management (IJAIEM), Volume 4, Issue 5, pp. 105-112, 2015

[18]. T.Subramani and M.Kavitha, “Analysis Of Reliability Of Steel Frame Systems With Semi-Rigid Connections Using Numerical Method And Finite Element Analysis", International Journal of Applied Engineering Research (IJAER), Volume 10, Number 38,Special Issues,pp.28240-28246, 2015.

[19]. T.Subramani, M.S.Saravanan, “Analysis Of Non Linear Reinforced And Post Tensioned Concrete Beams Using ANSYS”, International Journal of Applied Engineering Research (IJAER) International Journal of Applied Engineering Research (IJAER), Volume 10, Number 38 Special Issues, pp.28247-28252, 2015

[20]. T.Subramani, K.Balamurugan , "Finite Element Anaylsis Of Composite Element For FRP Reinforced Concrete Slab By Using ANSYS" , International Journal of Application or Innovation in Engineering \& Management (IJAIEM), Volume 5, Issue 5, pp. 076-084, 2016.

[21]. T.Subramani, V.KanianPoonkundran , " Prefabricated Multistory Structure Exposure To Engineering Seismicity By Using SAP" ,
International Journal of Application or Innovation in Engineering \& Management (IJAIEM), Volume 5, Issue 5, pp. 123-131, 2016.

[22]. T.Subramani, A.Kumaravel , " Analysis Of Polymer Fibre Reinforced Concrete Pavements By Using ANSYS" , International Journal of Application or Innovation in Engineering \& Management (IJAIEM), Volume 5, Issue 5, pp. 132-139, 2016

[23]. T.Subramani, R.Praburaj , " Pushover Anaylsis Of Retrofitted Reinforced Concrete Buildings By Using SAP" , International Journal of Application or Innovation in Engineering \& Management (IJAIEM), Volume 5, Issue 5, pp. 140-147, 2016.

[24]. T.Subramani, M.Senthilkumar , "Finite Element Anaylsis Of RC Beams With Externally Bonded Simcon Laminates By Using ANSYS" , International Journal of Application or Innovation in Engineering \& Management (IJAIEM), Volume 5, Issue 5, pp. $148-155,2016$

[25]. T.Subramani, R.Vasanthi , " Earth Quake Resistant Building Using SAP" , International Journal of Application or Innovation in Engineering \& Management (IJAIEM), Volume 5, Issue 5, pp. 173-181, 2016 .

[26]. T.Subramani, A.Selvam , " Studies On Economical Configuration Of RCC And Prestressed Shell Roofs By Using ANSYS ", International Journal of Application or Innovation in Engineering \& Management (IJAIEM), Volume 5, Issue 5, pp. 182-191, 2016.

[27]. T.Subramani, A.Anbuchezian , " Experimental Investigation On Flexural Behavior Of Folded Ferro Cement Panels " , International Journal of Application or Innovation in Engineering \& Management (IJAIEM), Volume 6, Issue 3, March 2017 , pp. 045 049 , ISSN 2319 - 4847.

[28]. T.Subramani, A. Fizoor Rahman , " An Experimental Study On The Properties Of Pet Fibre Reinforced Concrete " , International Journal of Application or Innovation in Engineering \& Management (IJAIEM), Volume 6, Issue 3, March 2017 , pp. 058066 , ISSN 2319 - 4847.

[29]. T.Subramani, S.Poongothai, S.Priyanka , " Analytical Study Of T Beam Column Joint Using FEM Software " , International Journal of Emerging Trends \& Technology in Computer Science (IJETTCS), Volume 6, Issue 3, May - June 2017 , pp. 148-156 , ISSN 2278-6856

[30]. T.Subramani, R.Ganapathy,V.Manoharan, M.Balamurugan, R.Murugesan , " Design And Analysis Of Light Weight Concrete Building Using ETAB With Respect To Dynamic Loading " , International Journal of Emerging Trends \& Technology in Computer Science (IJETTCS), Volume 6, Issue 3, May - June 2017 , pp. 252-258, ISSN 2278-6856.

[31]. T.Subramani, A.Mohammed Ali, R.Karthikeyan, E.PannerSelvan , K.Periyasamy, " Analytical Study Of T-Beam Using ANSYS " , International Journal of Emerging Trends \& Technology in Computer Science (IJETTCS), Volume 6, Issue 3, May - June 2017 , pp. 259-266, ISSN 2278-6856.

[32]. T.Subramani, V.Kalaivanan, S.Priyaranjithkumar, P.Sasikumar, P.Vinoth Kumar, " Design And Analysis Of Multistorey Building With Respect To Seismic Loads Using ETABS " , International Journal of Emerging Trends \& Technology in Computer Science (IJETTCS), Volume 6, Issue 3, May - June 2017, pp. 267-274, ISSN 2278-6856. 